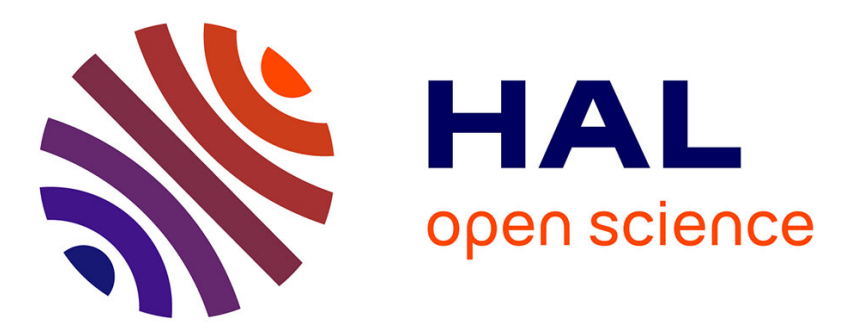

\title{
Transient Fission Matrix approach for assessing complex kinetics behavior in the ZEPHYR ZPR coupled core configurations
}

Patrick Blaise, Axel Laureau, Paul Ros, Pierre Leconte, Kornilios Routsonis

\section{- To cite this version:}

Patrick Blaise, Axel Laureau, Paul Ros, Pierre Leconte, Kornilios Routsonis. Transient Fission Matrix approach for assessing complex kinetics behavior in the ZEPHYR ZPR coupled core configurations. Annals of Nuclear Energy, 2019, 128, pp.390-397. 10.1016/j.anucene.2019.01.031 • cea-02510825

HAL Id: cea-02510825

https://hal-cea.archives-ouvertes.fr/cea-02510825

Submitted on 18 Mar 2020

HAL is a multi-disciplinary open access archive for the deposit and dissemination of scientific research documents, whether they are published or not. The documents may come from teaching and research institutions in France or abroad, or from public or private research centers.
L'archive ouverte pluridisciplinaire $\mathbf{H A L}$, est destinée au dépôt et à la diffusion de documents scientifiques de niveau recherche, publiés ou non, émanant des établissements d'enseignement et de recherche français ou étrangers, des laboratoires publics ou privés. 


\title{
Transient Fission Matrix approach for assessing complex kinetics behavior in the ZEPHYR ZPR coupled core configurations
}

\author{
Patrick Blaise $^{a l}$, Axel Laureau ${ }^{b}$, Paul Ros $^{c}$, Pierre Leconte $^{a}$, Kornilios Routsonis ${ }^{a}$ \\ a :CEA, DEN, DER, Cadarache, 13115 Saint-Paul-Lez-Durance \\ b : Ecole Polytechnique Fédérale de Lausanne (EPFL), \\ c : Institut de Radioprotection et de Sûreté Nucléaire (IRSN), Fontenay-aux-Roses, France
}

\begin{abstract}
The studies presented in this paper are performed in the general framework of future $3 D$ kinetics capabilities of the ZEPHYR (Zero power Experimental PHYsics Reactor) ZPR currently being designed at the CEA's Cadarache Centre. Transient calculations with accurate neutron kinetics models are required to characterize spatial decoupling in the cores that are expected to be built for reactor physics purposes. An innovative fission matrix interpolation model has been implemented with a correlated sampling technique associated to the Transient Fission Matrix (TFM) approach and applied to the ZEPHYR reference fast/thermal coupled configuration. These studies are performed in the general framework of transient coupled calculations with accurate neutron kinetics models. Depending on the targeted accuracy, feedback can be limited to the reactivity for point kinetics (PK), or can take into account the redistribution of the power in the core for spatial kinetics (SK). The local correlated sampling technique for Monte Carlo calculation presented in this paper was developed for this purpose, i.e. estimating the coupling coefficients of influence on the neutron transport of a local variation of different parameters on integral quantities such as $k$-eff. A dedicated $1 D$ benchmark demonstrates interesting features of this kind of coupled configurations for the validation of complex space-time kinetics transient experiments.
\end{abstract}

Key Words: Transient fission matrices, spatial kinetics, ZEPHYR fast/thermal coupled core.

\section{INTRODUCTION}

The study of power reactor behavior during normal and abnormal operation raises the incentive of modeling the transient phases. This kind of application may require Multiphysics able to take into account the interaction between the neutronics that provides the fission power source and other physics such as the thermal hydraulics that models the cooling aspects, or mechanics to take into account the core deformation or the pellet-cladding interaction. Within the multiphysics problem, complex 3D kinetics behavior of coupled cores are one of the most challenging problem to be solved in the near future, both for deterministic schemes, but also for newly developed and implemented methodologies in modern Monte Carlo codes such as TRIPOLI, Serpent, or MCNP. Several innovative MTR and technological demonstrator concepts include strong heterogeneities that increase the neutron gradient and consequently may induce a spatial decoupling between fissile zones during different transients potentially leading to accident scenarios. For this reason, spatial neutron kinetic models must be developed to verify that the flux redistribution remains limited in such situations. Dedicated appropriate physical models and numerical resolution to balance precision and reasonable computation time. In this frame, some simplifying assumptions in neutron kinetics modeling have to be made since the increase of computation capabilities is still not sufficient for direct time-dependent Monte Carlo calculations at the full reactor core scale. Hybrid approaches may be used, like improved quasistatic methods, but they require regular updates of the power shape and of the reactivity using precise core calculations.

\footnotetext{
${ }^{1}$ Corresponding author: patrick.blaise@cea.fr
} 
To this end, the spatial kinetic Transient Fission Matrix (called TFM) approach previously developed and presented in [1,2] has been adapted to model the effect of local medium perturbations [3]. The TFM approach is based on a conversion to discretized Green functions of the Monte Carlo response of the system in order to perform kinetic calculations without new reference calculation during the transient and thus with a reduced computation time. This approach is used to model neutron kinetics during transient coupled calculations; in order to take into account the evolution of the system during transient. TFM is associated to specific interpolation models that produce on the fly estimations of the matrix variations during the transient without requiring new Monte Carlo calculations. Such an interpolation model based on the correlated sampling technique and suitable for heterogeneous cores with a fast neutron spectrum has been developed. The resulting neutronics approach called 'perturbative TFM' is applied in this paper on the ZEPHYR fast/thermal coupled core concept $[4,5]$ currently being envisaged to become critical in Cadarache at the horizon 2028.

The present paper will describe the ZEPHYR ZPR project, and in particular its capacity to perform coupled core configurations for large 3D-kinetics transient applications. The TFM methodology is then briefly resumed before going to a direct application of its development on the fast/thermal (MOX$\mathrm{Na} / \mathrm{UO}_{2}-\mathrm{H}_{2} \mathrm{O}$ ) reference coupled core. The application on a simplified 1D problem will exhibit TFM capabilities to clearly point out coupling coefficients, as a preliminary way to design strong 3D transients in the forthcoming ZEPHYR coupled cores.

\section{THE ZEPHYR FACILITY}

Anticipating the decision to close both EOLE and MINERVE experimental facilities by the end of 2017, CEA started the design of a new versatile ZPR called ZEPHYR (Zero power Experimental PHYsics Reactor), a future facility to be built in Cadarache around 2028. Through a progressive optimization taking benefit from the MASURCA fast ZPR and its available fuel stockpile, the study aimed at answering recurrent questions about such configurations. The main goal of fast-thermal experimental programs is to measure integral total cross-sections of several isotopes of interest in a targeted fast spectrum through accurate reactivity effects measurements. Therefore, the neutronics characteristics of this spectrum have to be reproduced in the center of the fast-thermal coupled configuration. The work presented here is based on a PHENIX-like fast unit cell, called ZONA1, from ZONA1 core of the ERMINE V program [6] whose materials are coming from the MASURCA stockpile. ZONA1 cell is made of 6 enriched MOx rodlets, 2 natural UOx rodlets and 8 sodium pellets. It is 12 inches high and about 2 inches large. It represents an intermediate spectrum, between the two cores of PHENIX, and is represented below on Figure 1:

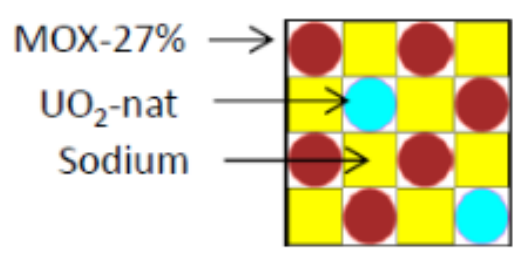

Figure 1: ZONA1 MOX unit cell

A first configuration is made of those ZONA1 cells, 36 inches high, that corresponds to the fissile height of the core. The radius is adapted in order to be critical. This entire fast configuration stands for the reference case and will stand as a target for both spectrum and reactivity effects to be reproduced in the mock-up. By coupling a center part of this reference configuration to a peripheral thermal zone, a basic coupled configuration is obtained. However, an adaptation zone was designed to optimize the target spectrum in the central channel of the experimental zone, using representativity approach. The fraction of delayed neutrons $\beta_{\text {eff }}$ and the effective neutron lifetime $\Lambda_{\text {eff }}$ have been calculated thanks to a dedicated module in the BISTRO code [7]. In the optimized configuration with a $\mathrm{B}_{4} \mathrm{C}$ ring, $\beta_{\text {eff }}$ is $742 \mathrm{pcm}$ whereas in the equivalent critical full fast reference configuration $\beta_{\mathrm{eff}}$ should be $403 \mathrm{pcm}$. This difference comes from the large proportion of Uranium isotopes. The effective neutron lifetime is $17 \mu \mathrm{s}$ in the optimized 
configuration with a $\mathrm{B}_{4} \mathrm{C}$ ring whereas its value in the fast reference configuration equals $0.3 \mu \mathrm{s}$. This value of $742 \mathrm{pcm}$ brings additional safety margins during the experimental programs while reducing the required amount of fast fissile materials to reach representative spectrum. A representation of the 3D geometry in plane (X-Y) obtained with a Monte-Carlo calculation by TRIPOLI-4® [8] is presented in Figure 2:

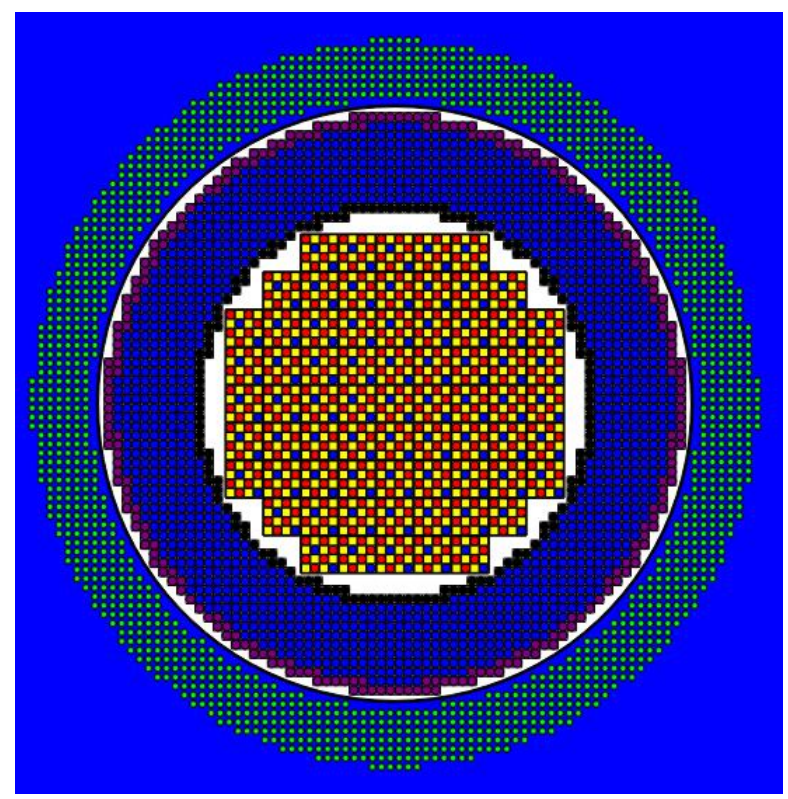

Figure 2: X-Y representation of the TRIPOLI4® modelling of the Fast/Thermal optimized configuration Blue : light water-Green : $U_{2} 3.7 \%$ enrichment - Purple : Metallic U $30 \%$ enrichment Navy : natural $\mathrm{UO}_{2}-$ Black : $\mathrm{B}_{4} \mathrm{C}$ absorber - White : Air The central cells are the one presented in Fig.1

The different areas in ZEPHYR are characterized by very different spectra as represented in Figure 3: 


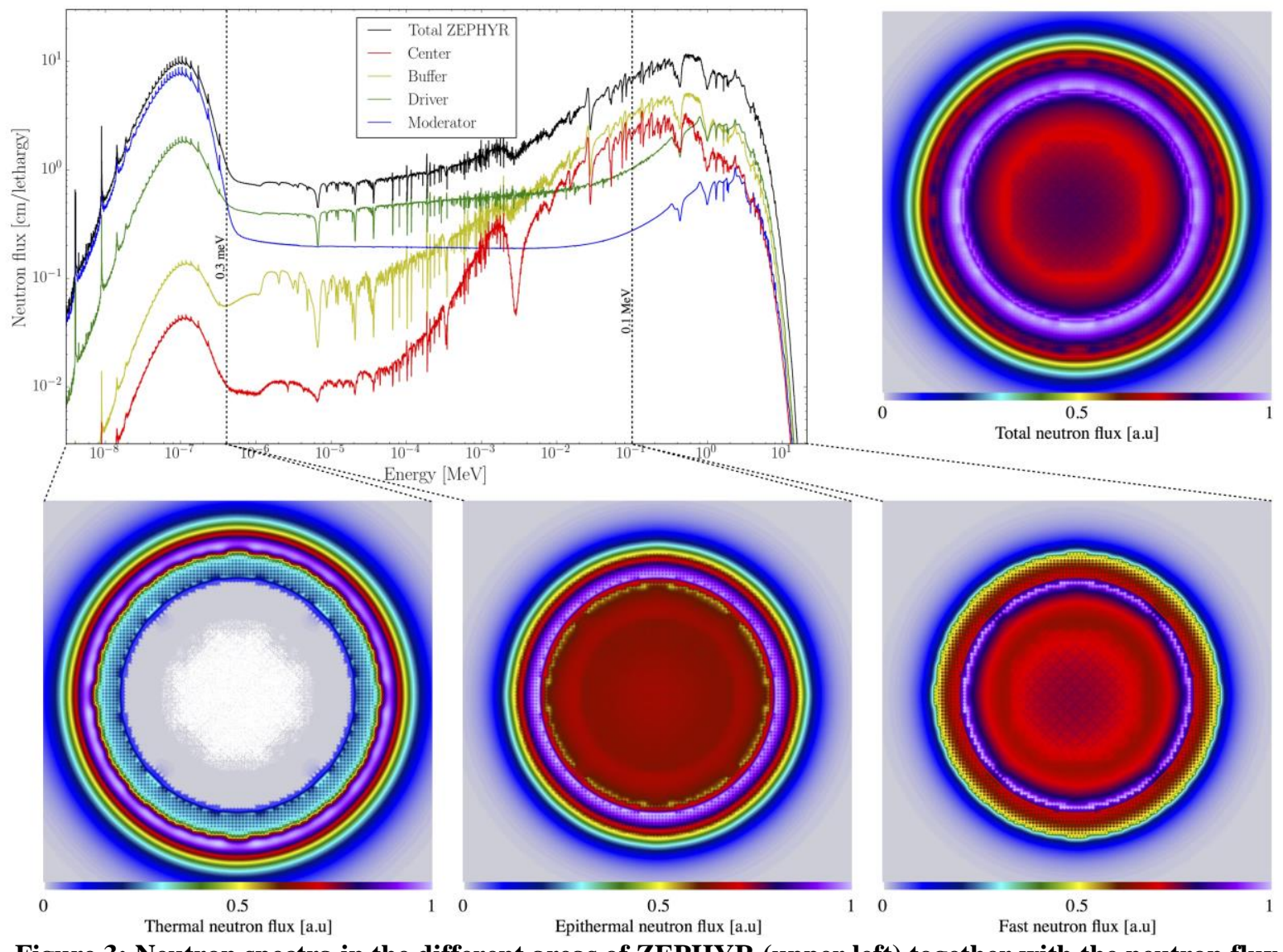

Figure 3: Neutron spectra in the different areas of ZEPHYR (upper left) together with the neutron flux maps calculated with the Serpent 2 code: total flux (upper-right), and thermal/epithermal/fast flux (bottom)

As several different zones are coupled, the radial profile of the adjoint flux along the core mid plane, reproduced in Fig. 4 is original and brings the information of two separate fissile zones. This effect will be pinpointed by the TFM approach.

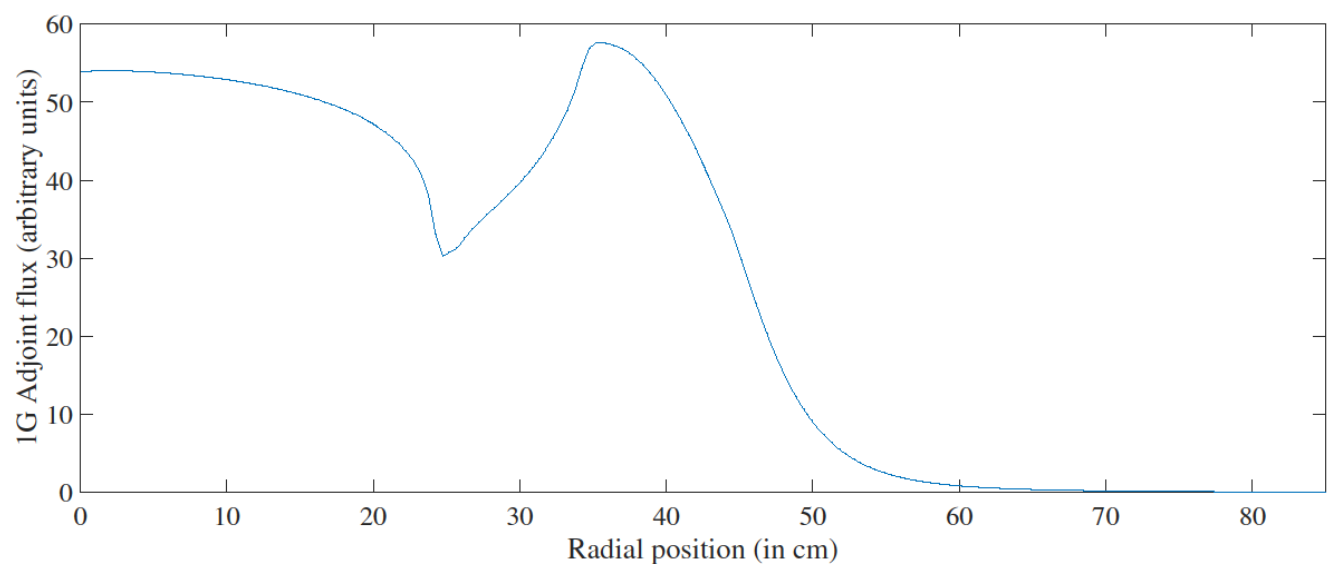

Figure 4: Radial representation of the $1 G$ adjoint flux in the ZEPHYR fast/thermal coupled configuration

This figure illustrates the importance of the high enriched Uranium ring and the decoupling effect of the transition zone around $\mathrm{R}=25 \mathrm{~cm}$. The TFM plots presented in section 4 will address these characteristics. 


\section{TRANSIENT FISSION MATRIX APPROACH}

The Transient Fission Matrix approach is a time dependent version of the fission matrices. Its objective is to precalculate the time dependent transport characteristics of the neutrons in a discretized geometry, in order to perform transient coupled calculations with a reduced calculation time. The raw information contained in the fission matrices $\underline{\mathbf{G}}$ is the probability that a fission neutron created in a volume $j$ produces a new fission neutron in a volume $i$. This information is then summarized in ad hoc formatted matrices (line $i$ column $j$ ), built according to either emission spectrum $\chi$ (prompt or delayed), or neutron multiplicity $v$ (prompt or delayed), e.g. $\underline{\mathrm{G}} \chi_{\mathrm{p}} v_{\mathrm{p}}$ as detailed below. In addition to the previous matrix construction average prompt propagation time from $j$ to $i$ can also be stored in the $\mathrm{T} \chi_{\mathrm{p}} v_{\mathrm{p}}$ time matrix (line $i$ column $j$ ). The present work extends this development to fast/thermal coupled core characteristics.

\subsection{TFM approach}

A clear and complete introduction of the usual fission matrix can be found in [1-3, 9, 10]. This tool is designed to characterize the neutron propagation in a reactor during one generation: the Green function is the system response to a neutron pulse. The objective of the present paper being to illustrate a concrete application to coupled core kinetics, interested people is welcomed to read the aforementioned references. Note that the final aim of this approach is not to produce a reference solution such as a direct kinetic Monte Carlo calculation, but to provide a precise spatial kinetic modeling with a reasonable computation time.

\subsubsection{Prompt and delayed neutrons}

In order to perform transient calculations using the fission matrices, two pieces of information have to be added to the usual fission matrices: the distinction between delayed and prompt neutrons and the temporal aspect. Prompt (labelled $p$ ) and delayed (labelled $d$ ) neutrons have different emission spectra $\chi_{\mathrm{p}}$ and $\chi_{\mathrm{d}}$ and consequently distinct behaviors in the reactor. Moreover, the production of delayed neutrons differs from that of prompt neutrons as their multiplicities $v_{\mathrm{p}}$ and $v_{\mathrm{d}}$ are different. For these reasons, four different matrices have to be calculated to take into account each case: $\underline{\mathrm{G}} \chi_{\mathrm{p}} v_{\mathrm{p}}, \underline{\mathrm{G}} \chi_{\mathrm{p}} \nu_{\mathrm{d}}$, $\underline{\mathrm{G}} \chi_{\mathrm{d}} \nu_{\mathrm{p}}$ and $\underline{\mathrm{G}} \chi_{\mathrm{d}} \nu_{\mathrm{d}}$. Most important phenomena for understanding coupling phenomena are the promptto-prompt and prompt-to-delayed matrices.

They correspond to the transport probabilities using their respective emission spectrum $\chi$ and neutron production multiplicity v. Calculations are performed with a Monte Carlo neutronic code using the same approach as for the usual fission matrices with two additional aspects: a link to the type of the neutron at it's birth (prompt or delayed) and also to the type of the created neutrons. For example the $(i, j)$ element of the $\underline{\mathrm{G}} \chi_{\mathrm{d}} v_{\mathrm{p}}$ matrix contains the probability that a prompt neutron emitted in the $j$ volume creates a new delayed neutron per fission in the $i$ volume. This estimation is done during a 'classic' criticality Monte Carlo calculation.

\subsubsection{Temporal aspect}

The second factor required to perform neutron kinetics with the fission matrices is the temporal aspect of the neutron propagation. Considering that the neutron transport time is negligible compared to the delayed neutron precursor lifetime, only the prompt neutron fission to fission time matrix $\underline{T} \chi_{\mathrm{p}} v_{\mathrm{p}}$ is needed (delayed neutrons are directly produced after the precursor decay). This matrix contains the average propagation time for a source neutron from $j$ to $i$, also evaluated in the same calculation as the matrices.

\subsubsection{Effect of material modification}

In order to deal with possible feedback effects during transient calculations, such as temperature (through Doppler broadening or thermal expansion of materials), dedicated interpolation models are required to take into account the modification of the matrices. Previous studies [9-10] shown that for a 
reactor with a small migration length (such as a PWR) or with homogeneous areas (such as the liquid fuel reactor MSFR), an interpolation based on reference and perturbed matrices calculations is enough if a capture matrix is added to properly normalize (to one) the number of neutron captures per propagated neutron. However, as shown on Fig.3 and Fig.4, the ZEPHYR reactor is characterized by strong local variations of direct and adjoint neutron spectra and amplitude. For this reason, a more generic interpolation initially developed in [1] for the heterogeneous ASTRID reactor was used and applied for material density modification, Doppler broadening feedback or concentration variations.

\subsection{Calculation parameters}

\section{METHODOLOGY AND TOOLS}

The TFM matrices are calculated with a modified version of Serpent 2.1.21 [11]. The modifications concern the calculation of the fission matrices, including the distinction between prompt and delayed neutrons, the fission to fission time matrix, and the correlated sampling technique to generate the locally perturbed matrices. The nuclear database used is JEFF 3.1 [12]. The number of simulated neutrons for each calculation is one billion.

\subsection{ZEPHYR 1D Simplified Model}

The preliminary analysis is performed of the optimized fast/thermal configuration designed in ZEPHYR for fast/thermal coupled configuration, and reproduced on Figures 5. In order to test the TFM approach, a simplified 1D benchmark is proposed for the sake of the study. The system boundary conditions for the $1 \mathrm{D}$ is void on radial boundaries in order to reproduce leakage outside the lattice, and reflexion on the axial boundaries.

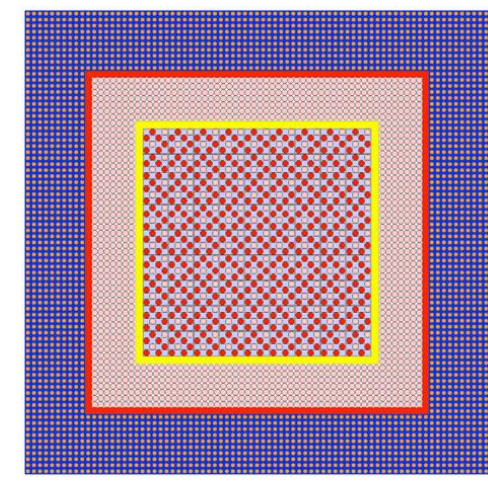

Figure 5a: squared 2D

representation of the ZEPHYR optimized fast/thermal coupled configuration
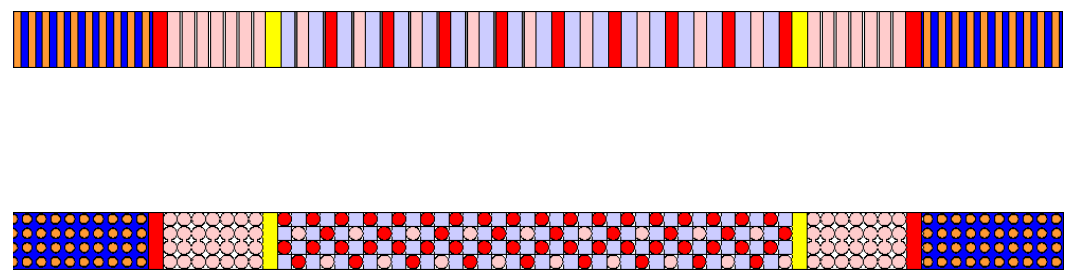

Figure 5b: 1D Traverse of the optimized fast/thermal coupled configuration

Figure 5a describes the 2D squared geometry from whom the 1D benchmark is extracted (Fig. 5b). Periodicity between the two other axes ensures the 1D characteristics of this model.

The geometry is splitted in 74 meshes as follows :

- Thermal zone: meshes 1 to 10 (and 65 to 74 by symmetry)

- Conversion zone: mesh 11 (and 64 by symmetry)

- Transition zone: meshes 12 to 19 (and 56 to 63 by symmetry) constituted by nat $\mathrm{UO}_{2}$ and $\mathrm{B}_{4} \mathrm{C}$ (meshes 19 and 56)

- So-called "ZONA1" cell zone: meshes 20 to 55

The transient calculation is performed by calculating, for each node, prompts and delayed contributions. 


\section{CALCULATION RESULTS}

\subsection{Transient fission matrices}

Four (4) fission matrices are available, translating the probability that a prompt neutron born in mesh $j$ induces a delayed neutron in $i$ for the "prompt to delayed" TFM. The most significant matrix, as it contains the higher number of neutrons, is the «prompt to prompt » reproduced in Fig 6.

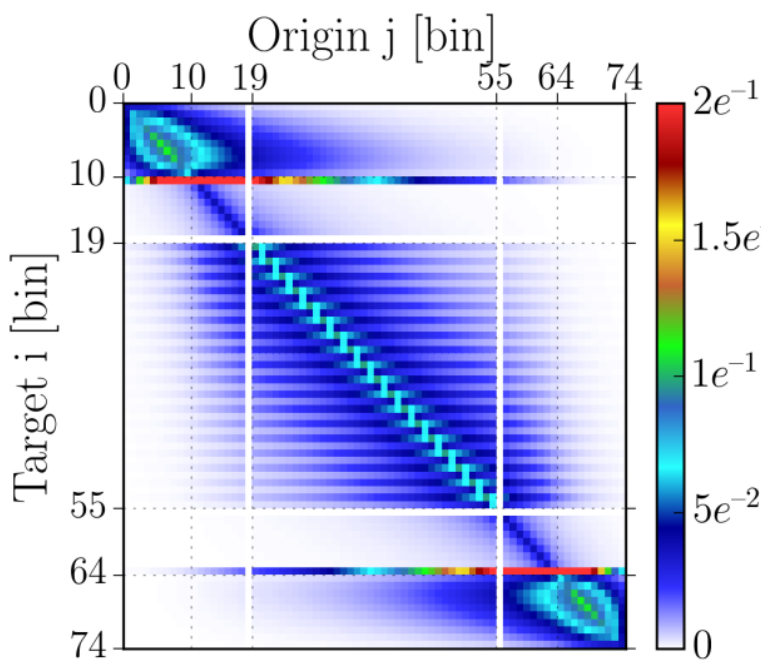

Figure 6: « prompt-to-prompt » fission matrix

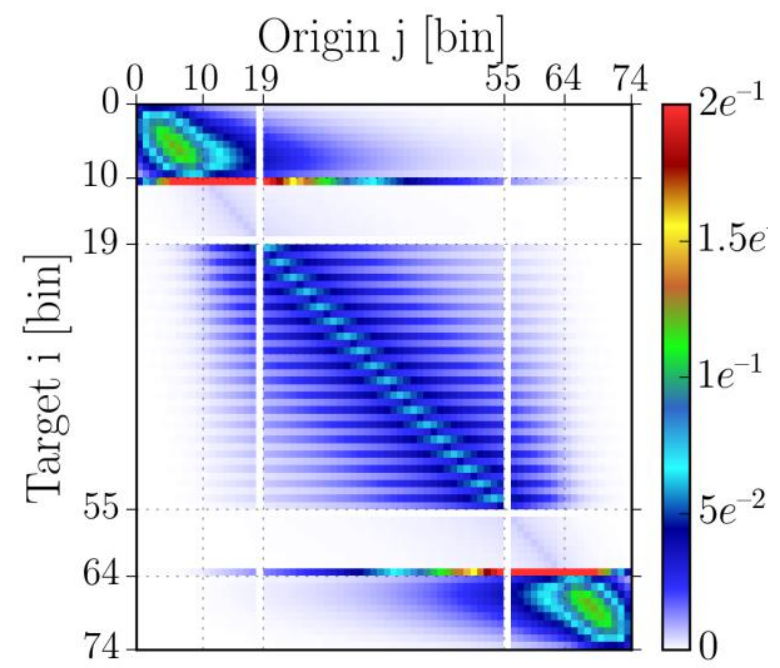

Figure 7: « delayed-to-prompt » fission matrix

All diagonal terms present important probabilities: numerous fissions appear close to the emission of the "father" neutron. This is even more exhibited in thermal zones where the diagonal elements are even tighter, being a consequence of shorter neutron mean free paths. The case of metallic uranium is interesting as being non-symmetrical. The $U_{\text {met }}$ fuel is the source of mainly all fission neutrons (mesh 11). Few fission appear either in the fast zone, or in the thermal buffer zone. Starting from column 11, almost no neutron in the central meshes is produced from initial neutron from the conversion zone. Hence, the "prompt to prompt" matrix mostly points out strongly ... decoupled cores.

Looking carefully to fast/thermal decoupling, it is noteworthy to observe that the probability to generate fissions in the core center is very low (less than 1\%), except for fission neutrons coming from a fission into a ZONA1 cell. If the probability to generate a fission neutron in the center from a neutron born in the nat- $\mathrm{UO}_{2}$ is not negligible, it has to be weighted by the low proportion of neutrons born in this area of the core. The adjoint transport operator, being a picture of the adjoint flux (see Fig 4), can be extracted from a column lecture for each line. Considering fission in a given mesh, the associated columns give the origin of the fission neutron, hence the importance of this neutron. For example, a lecture of line $i=37$ shows that fission neutron produced in the core center are mainly issued from the ZONA1 cells.

The decoupling is clearly observed on the $1 \mathrm{D}$ traverse of the prompt to prompt matrix eigenvector (Fig.8). At the periphery, the eigenvector follows a thermal flux shape (zone 1), with a peak corresponding to the enriched uranium rods (zone 2). After the adaptation zone (3), characterized by a very low importance (hence low eigenvector), the neutron flux stabilizes on the fast domain with a flat cosine shape (4). 


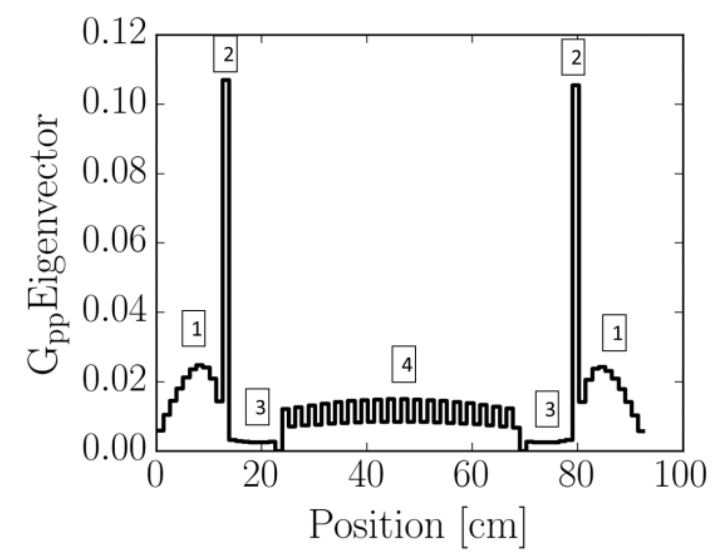

Figure 8: «prompt-to-prompt » fission matrix eigenvector

The other fission matrices linked to delayed neutrons are presented in the next figures (9 to 10). A qualitative interpretation of their particularities is made. The main difference between those matrices and the prompts ones comes from meshes 12 to 18 (natural $\mathrm{UO}_{2}$ ) where emission probabilities tend to zero. This particular behavior mainly comes from dephasing between delayed neutron spectra, whose average energy is lower than the prompts neutrons, and the "fertile" nature of the medium, mainly composed of ${ }^{238} \mathrm{U}$. In fact, the average energy of the delayed "father" neutrons is much lower than the $1 \mathrm{MeV}$ fission threshold (average energy is around 300-400keV), and hence do not produce fissions.

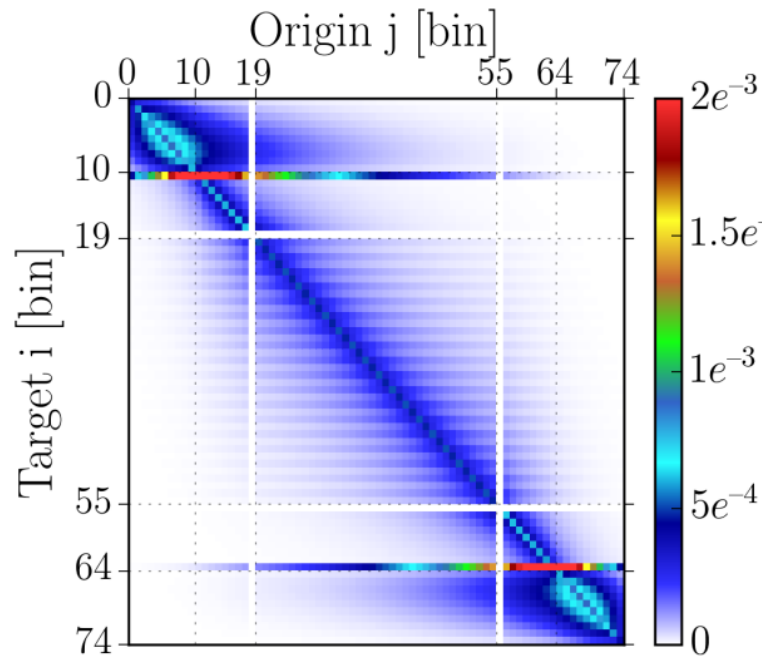

Figure 9: « prompt-to-delayed »fission matrix

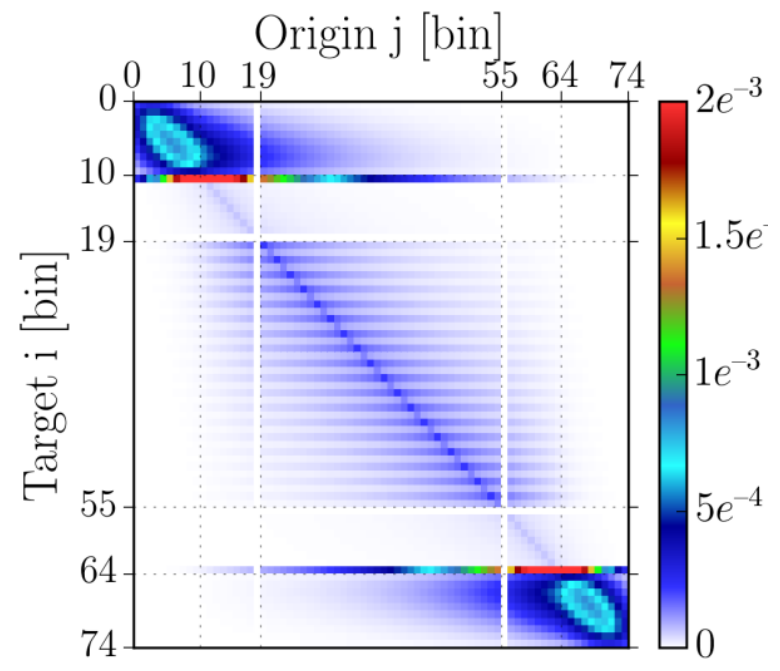

Figure 10 : « delayed-to-delayed » fission matrix

Delayed neutrons population being much lower than prompt neutrons population, the associated probabilities are of smaller amplitude. Throughout isotopes present in the core, uranium isotopes present the highest delayed neutron fractions. Again, one observes higher probabilities between meshes 12 to 18 , thanks to the presence of ${ }^{238} \mathrm{U}$. Inversely, the spread around the main diagonal is more restricted than previously, in particular around the ZONA1 cells, due to a lower delayed neutrons fraction of the plutonium isotopes.

Figure 10 mainly focuses on delayed neutrons fission matrix. It presents very weak probabilities in the core center, mainly due to the presence of plutonium isotopes, as well as « holes » in natural $\mathrm{UO}_{2}$ thanks to non correspondence between the main delayed neutron spectrum energy (few hundreds of $\mathrm{keV}$ ) and the ${ }^{238} U$ threshold fission cross section. In conversion and thermal zones, high values are obtained, as those zones are mainly composed of enriched uranium material. 
Figure 11 reproduces the $\mathrm{T} \chi_{\mathrm{p}} v_{\mathrm{p}}$ time operator of the «prompt to prompt » events. Average prompt neutrons lifetimes present strong differences and extend to two decades. This extension is due to the coupling of thermal and fast zones, through strongly different spectra.

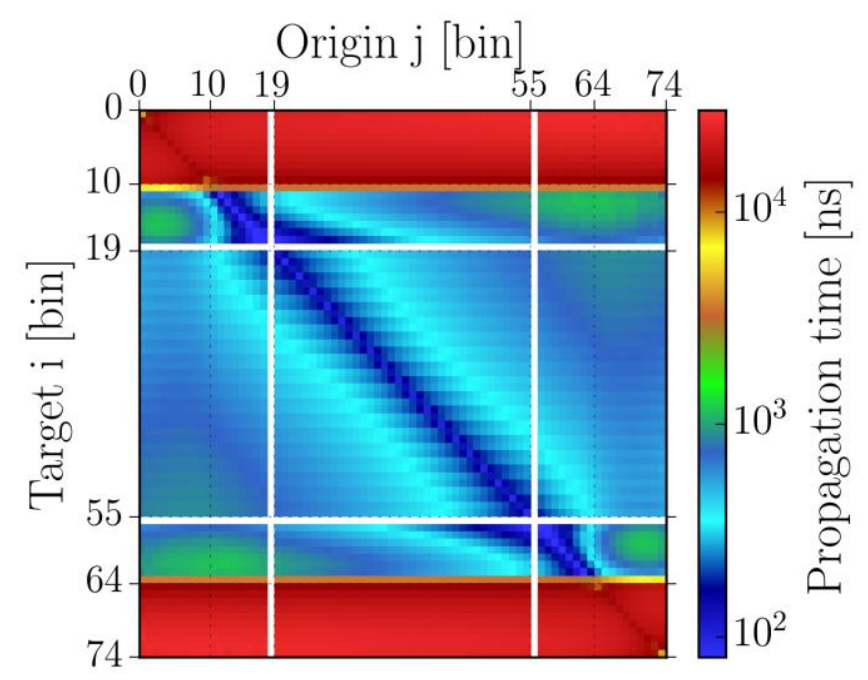

Figure 11: "prompt to prompt" time operator matrix

The correlation between spectrum and neutron lifetime is obvious neutrons become thermal through a time expensive multiple scattering process.

This matrix also points out that neutrons from the thermal zone and fissioning in the thermal zone, as well as neutrons from the fast zone which will produce a fission in the thermal zone are characterized by the largest lifetime (above $10 \mu \mathrm{s}$ ) : they are seen by red bands on the upper and lower parts (arrival meshes 1 to 10). Within the fast zone (meshes 12 to 64), neutron lifetimes are shorter as neutrons issued from the fast zone induce fission as they are still fast. However, one can observe a slight difference in mesh 11, where neutrons from the ZONA1 zone have to go through the whole conversion zone to induce fission in the enriched metal uranium. Neutrons issued from the thermal zone and making a fission in the central fast zone (originating from meshes 1 to 10 and 65 to 74 ) have a low lifetime : only fast neutrons from the thermal zone are able to cross the conversion zone.

\subsection{Material perturbation and impact on the fission matrices}

Local and global perturbations can also be treated directly through TFM approach. We will here after give two examples related to sodium rodlets present in the geometry. The first one illustrates the effect on a global perturbation of the sodium density of $1 \%$, captured by both correlated sampling and independent Monte Carlo calculation. The perturbation on the probability is plotted on figure 12 . 

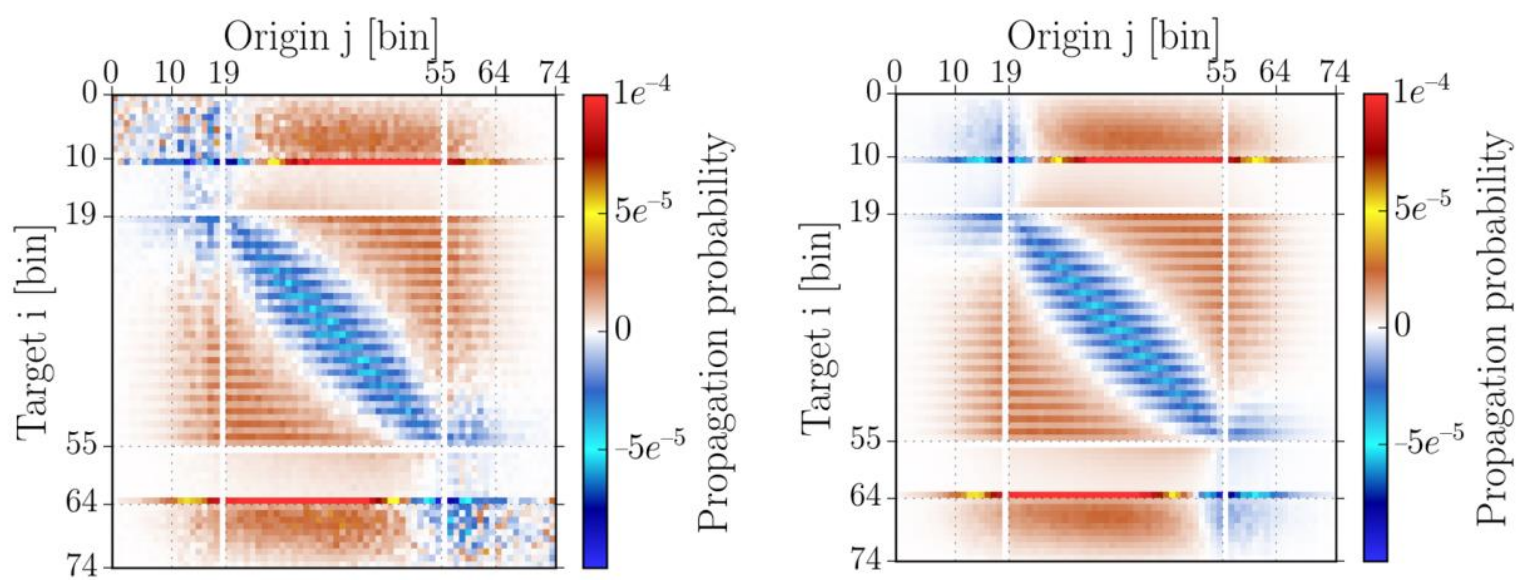

Figure 12: " vp $\rightarrow \chi p$ " discrepancy between two states by independant Monte Carlo approch (left) and correlated sampling (right)

Several observations can be made:

- The correlated sampling approach exhibits better convergence,

- The propagation probability is reduced on the main diagonal, meaning that the fission probability is lower than in the reference case. In fact, the phenomenon is linked to the higher mean free path of neutrons propagating in less dense medium. When sodium density is decreased, the neutrons scatter further in the geometry, hence locally reducing the fission probability.

The local perturbation effect of the sodium density can be plotted. Figure 13 reproduces a perturbation of the sodium density close to the fast/thermal interface.

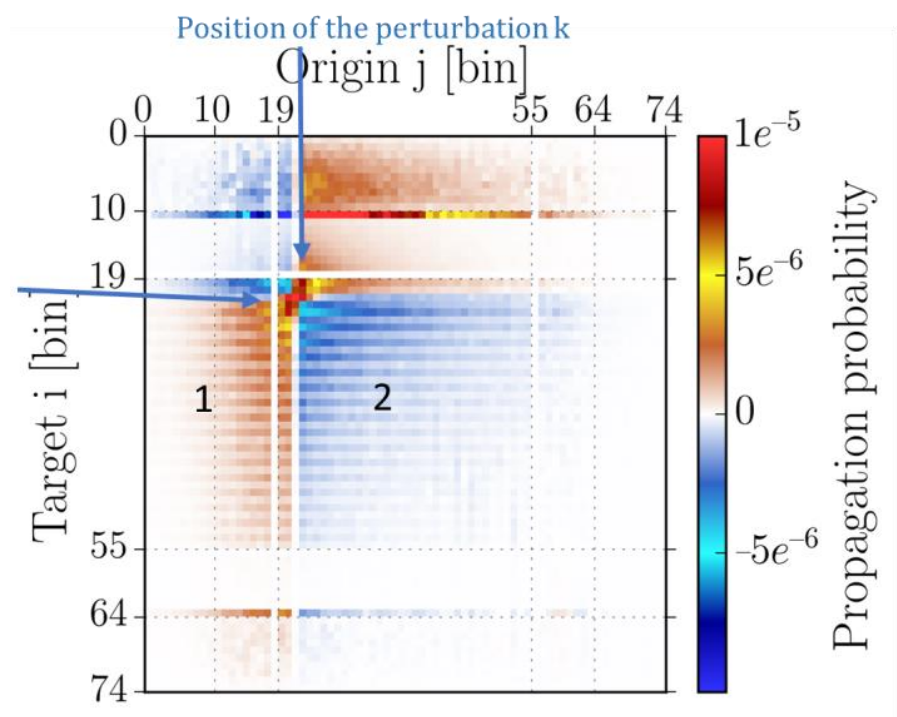

Figure 13: local impact of a $1 \%$ perturbation in the sodium density on the fission probabilities

Several zones $(1 \& 2)$ around the perturbation must be explained.

Case 1: if the position of the perturbation $k$ is between the emission position $j$ and the fission position $i$ $(i<k<j)$ or- $(j<k<i)$, then the neutron crosses the perturbation and undergoes a local spectrum hardening (leading to more fissions in the MOX) in $k$, as its mean free path increases due to the density decrease. 
Case 2: if $(i$ and $j)<k$ or $(i$ and $j)>k$, then the proportion of neutrons having crossed the perturbed area having less probability to scatter back, the fission probability decreases accordingly in $j$ and $i$.

Figure 14 reproduces the evolution of the local perturbation as it crosses the geometry. This perturbation displacement is easily illustrated by plotting the radial sensitivity profile of the ZEPHYR benchmark eigenvalue, also reproduced on Figure 13.
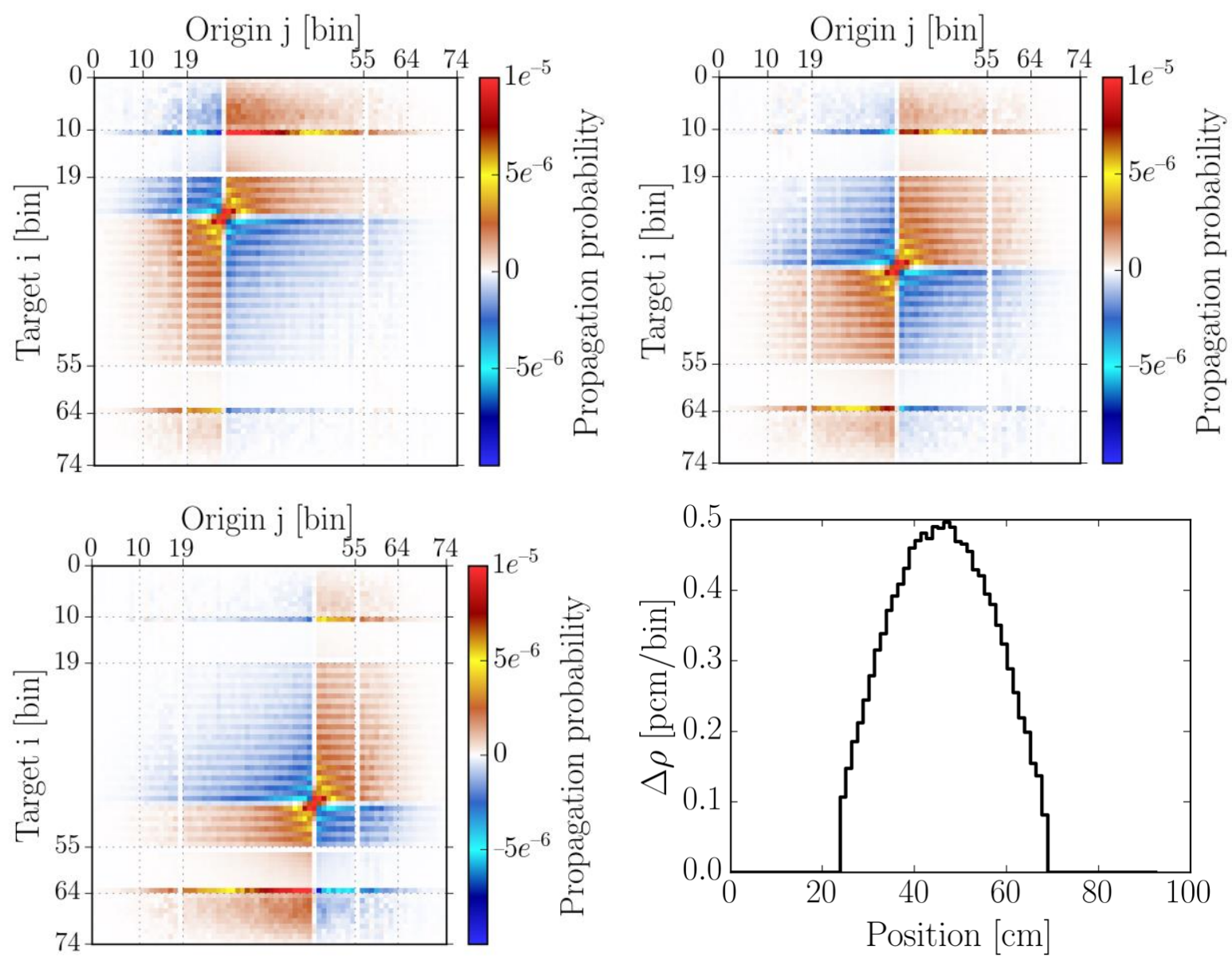

Figure 14: evolution of the fission matrix as the density perturbation crosses the geometry. The sensitivity profile of the reactivity per perturbed bin is also reproduced (lower right)
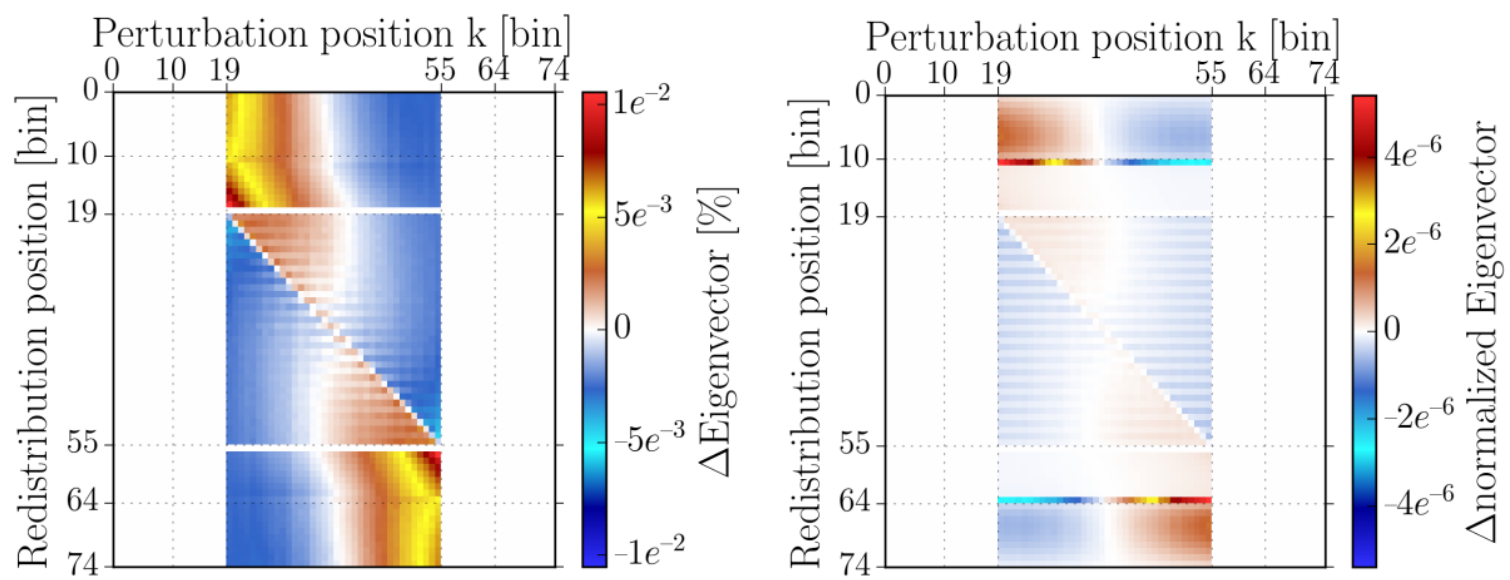

Figure 15: Eigenvector matrix redistribution due to local Na perturbation in bin 34. Left: perturbation on the eigenvector/bin (in \%) - Right: normalized perturbation 
Figure 15 depict an example of the eigenvector perturbation. Each column represent the eigenvector perturbation due to a local Na perturbation. The eigenvector-perturbed matrix exhibits a clear trend to bring back the fissions to the perturbed side, with a strong local discontinuity where the perturbation occurs.

\section{CONCLUSIONS}

A TFM approach was applied on a simplified coupled core 1D benchmark. Fission matrices as well as the temporal operator matrix are full of information for the purpose of the future ZEPHYR ZPR. They enable the physicist to qualitatively illustrate neutron coupling or decoupling effects between zones in strongly heterogeneous configurations, characterized by large delayed neutron fractions. Used as a complement to the coupling theories of Avery [13] and Kobayashi [14], they are a powerful tool for the physical analysis of complex transients. An extension of the TFM approach will be used in the near future to enlarge the ZEPHYR capabilities for exhibiting large coupling/decoupling effects, with the aim to build dedicated programs for full 3D transient programs in high dominant ratio geometries. In particular, the TFM will be implemented in the TRIPOLI-4@ MC code, and compared to multipoint approaches to solve complex 3D kinetics problems, as flux tilts in large cores.

\section{REFERENCES}

[1] Laureau, L. Buiron, B. Fontaine, "Local correlated sampling Monte Carlo calculations in the TFM neutronics approach for spatial and point kinetics applications", EPJ Nuclear Sci. Technol. 3, 16 (2017)

[2] A. Laureau, L. Buiron, B. Fontaine, "Towards spatial kinetics in a low void effect sodium fast reactor: core analysis and validation of the TFM neutronic approach", EPJ Nuclear Sci. Technol. 3, 17 (2017)

[3] A. Laureau, L. Buiron, B. Fontaine, V. Pascal "Fission Matrix Interpolation for the TFM Approach Based on a Local Correlated Sampling Technique for Fast Spectrum Heterogeneous Reactors", Proc. Int.Conf. M\&C 2017, Jeju, Korea, April 16-20, 2017

[4] P. Blaise, F. Boussard, P. Ros, P. Leconte, M. Margulis, G. Martin, K. Blandin "Experimental $R \& D$ innovation for Gen-2,3 \& IV neutronics studies in ZPRs: a path to the future ZEPHYR facility in Cadarache", Proc. Int. Conf IGORR, Berlin, March 2016

[5] P. Ros, P. Blaise, P. Leconte, H. Doger de Spéville, M. Maillot "Fast-thermal coupled cores in ZPRs : a demonstration of feasibility and pertinence for the ZEPHYR project", Ann. Nucl. Energy, 110, 290-305, December 2017

[6] P. Ros, P. Leconte, P. Blaise "Integral cross sections of fission products in fast reactors. Reinterpretation of the ERMINE V experiment : Interest of Fast-Thermal Coupled Cores", Proc. Int. Conf ND2016, Brugges, Sept 2016

[7] G. Rimpault, et al. "The ERANOS code and data system for fast reactor neutronic analyses", PHYSOR2002 Conference Seoul Korea 2002

[8] E. Brun, F. Damian, C. Diop, E. Dumonteil, F. Hugot, C. Jouanne, Y. Lee, F. Malvagi, A. Mazzolo, O. Petit et al., Tripoli-4®, CEA, EDF and AREVA reference Monte Carlo code, Ann. Nucl. Energy 82, 151 (2015)

[9] A. Laureau, Développement de modèles neutroniques pour le couplage thermohydraulique du MSFR et le calcul de paramètres cinétiques effectifs, Ph.D. thesis, Université Grenoble Alpes, 2015

[10] A. Laureau, D. Heuer, E. Merle-Lucotte, P. Rubiolo, M. Allibert, M. Aufiero "Transient coupled calculations of the Molten Salt Fast Reactor using the Transient Fission Matrix approach", Nuclear Engineering and Design, 316, 112-124, 2017

[11] J. Leppänen, M. Pusa, T. Viitanen, V. Valtavirta, T. Kaltiaisenaho, The Serpent Monte Carlo code: status, development and applications in 2013, Ann. Nucl. Energy 82, 142 (2015) 
[12] A.Santamarina et al. "The JEFF-3.1.1 Nuclear Data Library", JEFF Report 22, OCDE (2009)

[13] R. Avery, "Theory of coupled reactors", Proc. 2nd United Nations Conf. for Peaceful Use of Atomic Energy, United Nations, Vol. 12, p. 182 (1958)"

[14] Kobayashi "A relation of the Coupling coefficient to the eigenvalue separation in the coupled reactors theory", Ann. Nucl. Energy, Vol. 25, No. 4-5, pp. 198-201 (1998) 